\title{
Physical activity moderates the effect of sedentary time on an older adult's physical independence
}

\author{
Megan Hetherington-Rauth $\mathbf{P h D}^{\mathbf{1}} \quad \mid \quad$ João $\mathbf{P}$. Magalhães $\mathbf{P h D}^{\mathbf{1}}$ | \\ Pedro B. Júdice $\mathbf{P h D}^{2}$ | Ignacio Ara $\mathbf{P h D}^{3,4}$ | Gil B. Rosa MS ${ }^{\mathbf{1}}$ | \\ Inês R. Correia MS ${ }^{1} \quad$ Asier Mañas MS $^{3,4}$ | | Luís B. Sardinha $\mathbf{P h D}^{\mathbf{1}}{ }^{\circ}$
}

${ }^{1}$ Exercise and Health Laboratory, CIPER, Faculty of Human Kinetics, University of Lisbon, Lisbon, Portugal

${ }^{2}$ CIDEFES-Centro de Investigação em Desporto, Educação Física e Exercício e Saúde, Universidade Lusófona, Lisbon, Portugal

${ }^{3}$ GENUD Toledo Research Group, University of Castilla-La Mancha, Toledo, Spain

${ }^{4}$ CIBER of Frailty and Healthy Aging (CIBERFES), Madrid, Spain

\section{Correspondence}

Megan Hetherington-Rauth, PhD, Estrada da Costa, 1499-002, Cruz-Quebrada, Portugal.

Email: mchr@email.arizona.edu

\section{Funding information}

Fundação para a Ciência e a Tecnologia, Grant/Award Numbers: SFRH/

BD/149394/2019, UIDB/00447/2020

\begin{abstract}
Background/objectives: Moderate-to-vigorous physical activity (MVPA) and breaks in sedentary time (BST) have been proposed as viable solutions to improve an older adult's physical independence, whereas sedentary time (ST) has been associated with detrimental effects. We sought to assess the joint effects of ST, BST, and MVPA on the physical independence of older adults and determine whether and to what extent the ST relationship with physical independence is moderated by MVPA and/or BST.

Design: Cross-sectional.

Setting: Laboratory of Exercise and Health, Faculty of Human Kinetics.

Participants: Older adults ( $\geq 65$ years old) from the national surveillance system in Portugal $(n=821)$.
\end{abstract}

Measurements: Physical activity and ST were assessed by accelerometry. Physical independence was assessed using a 12-item composite physical function (CPF) questionnaire. Multiple linear regression was used to model the outcomes.

Results: Higher ST was related to lower CPF score $(\beta=-0.01, p<0.0001)$, whereas higher MVPA was related to better CPF score $(\beta=0.02, p<0.0001)$. BST was not related to physical independence after accounting for MVPA and ST $(\beta=0.03, p=0.074)$. MVPA had a moderating effect on the relationship of ST with CPF score $(p<0.0001)$, where MVPA $\geq 36.30 \mathrm{~min} /$ day ameliorated the significant inverse relationship between ST and CPF. Engaging in $\geq 107.78$ of MVPA resulted in ST having a significant positive relationship with CPF score. No moderation effect was found for $\operatorname{BST}(p>0.05)$.

Conclusion: Regardless of the time spent in MVPA and BST, ST was inversely related to CPF. However, MVPA was found to be a moderator of the relationship between ST and physical independence, such that engaging in at least $36 \mathrm{~min} /$ day of MVPA may blunt the negative effects of ST. At high levels of MVPA ( $\geq 108 \mathrm{~min} /$ day), having some ST may actually provide some benefit to an older adult's ability to maintain physical independence.

KE Y W O R D S

activities of daily living, aging, function, sedentary breaks, sedentary behavior 


\section{INTRODUCTION}

Having a physically active lifestyle is considered a key strategy to prevent the loss of physical independence and promote a healthy aging process in older adults. ${ }^{1,2}$ Beyond physical activity, epidemiological and experimental evidence have also identified sedentary time (ST) as a predisposing risk factor for health outcomes related to functional disability, reduced functional capacity, and frailty in older adults, ${ }^{3,4}$ independent of time spent in moderate-to-vigorous PA (MVPA). In fact, several investigations have highlighted the importance of interrupting the time spent in sedentary behavior by performing regular breaks of $\mathrm{PA}^{5-9}$ to improve the physical independence and function of older adults.

Much of the prior literature has not examined the joint and interacting effects of MVPA and breaks in sedentary time (BST) with ST. Thus, the question of whether individuals can overcome the detrimental effects of ST if they undertake higher amounts of MVPA or BST remains to be fully answered. It has recently been reported that $27 \mathrm{~min} /$ day of MVPA were needed to offset the harmful effects of ST on frailty in older adults, ${ }^{10}$ and between 30 and $40 \mathrm{~min} /$ day of MVPA were needed to eliminate the increased risk of premature death associated with ST in middle to older aged adults. ${ }^{11}$ Currently, the extent to which MVPA and/or BST may influence the effect of ST on the risk of physical dependence in older adults has not been investigated.

The aims of this investigation were twofold: (1) assess the joint effects of objectively measured ST, BST, and MVPA on the physical independence of older adults; and (2) to determine if the relationship of ST with physical independence is moderated by MVPA and/or BST.

\section{METHODS}

\section{Participants}

This cross-sectional investigation included a total of 2666 older adults ( $\geq 65$ years old) who took part in a national survey examining a representative sample of the Portuguese population collected between March 2017 and November 2018. All of the participants were required to have the ability to perform activities of daily living independently. The study was implemented in full compliance with the Helsinki Declaration and approved by the University ethics committee (ID number: 25/2020). Written informed consent was obtained from all participants.

\section{Anthropometric assessments}

Weight and height were measured according to standardized procedures. ${ }^{12}$

\section{Key Points}

- The negative association between sedentary time (ST) and an older adult's physical independence was not significant when moderateto-vigorous physical activity (MVPA) was $>36 \mathrm{~min} /$ day.

- A positive association between ST on physical independence was found at an MVPA level > $108 \mathrm{~min} /$ day.

- The number of breaks in ST (BST) was not a moderator of the relationship between ST and an older adult's physical independence.

\section{Why Does this Paper Matter?}

This study shows that performing $\geq 36 \mathrm{~min}$ of MVPA per day can offset the detrimental effects of ST on an older adult's physical independence. Thus, public health policies should primarily target strategies to improve levels of MVPA and, secondly, reduce ST, to promote a healthy aging process.

\section{Socioeconomic demographics}

Socioeconomic variables included were age, sex, education level, monthly income, and marital status.

\section{Physical independence assessment}

Physical independence was assessed through self-report using the 12-item composite physical function (CPF) scale, ${ }^{13}$ where a higher $\mathrm{CPF}$ score indicated a greater degree of physical independence.

\section{Physical activity and sedentary time assessments}

Physical activity was assessed using an accelerometer (ActiGraph, GT3X+ model; Fort Walton Beach, FL). Participants were asked to wear the accelerometer on the right hip, close to the iliac crest, for seven consecutive days. Data were recorded at a $100 \mathrm{~Hz}$ frequency, and downloaded into $60 \mathrm{~s}$ epochs. Troiano et al. cutpoints and validation criteria were used to analyze the data. ${ }^{14} \mathrm{~A}$ BST was considered as an interruption of ST with $\geq 1$-min duration, in which the counts registered by the accelerometer rose above the threshold of 99 counts $/ \mathrm{min}$. 


\section{Statistical analysis}

All statistical analyses were performed using SPSS 25 (SPSS Inc., an IBM Company, Chicago, IL) and STATA 13.1 (StataCorp LLC, College Station, TX) using a significance level of $p<0.05$.

Sample characteristics were described using mean \pm SD and median (interquartile range) for normally distributed and skewed variables, respectively. Multiple linear regression was used to assess the associations between ST, MVPA, and BST with CPF score, while controlling for total accelerometer wear time, age, sex, and body mass index (BMI). Moderation of the relationship between ST and CPF score by BST and MVPA was assessed using separate regression models with the inclusion of an interaction term (ST*BST or ST*MVPA). When a significant interaction was observed, the Johnson-Neyman technique was used to assess at what values of the moderator (MVPA or BST) the effect of ST on CPF score transitioned from being significant to non-significant. A heteroscedastic consistent standard error estimator (DavidsonMackinnon) was applied to all models given the observed heteroscedasticity of the model residuals.

\section{RESULTS}

Out of the 2666 older adults assessed, 821 (aged 75.5 \pm 7.4 years-old) had complete data on PA, ST, and CPF score and were included in the analyses (Table 1). Older adults excluded from the analysis were slightly older and were of lower socioeconomic status compared to the included sample, but did not differ in terms of sex distribution or BMI. The median MVPA was $9.3 \mathrm{~min} /$ day, with $70 \%$ of the sample not meeting the PA guidelines of at least $21.4 \mathrm{~min} /$ day of moderate PA. ${ }^{15}$

Higher ST was related with lower CPF values $(\beta=-0.01, p<0.001)$, such that spending $60 \mathrm{~min} /$ day more in ST translated to a 0.6 unit lower CPF score (Table 2). On the contrary, greater levels of MVPA were related to better CPF score (MVPA $\beta=0.02, p<0.008$ ), such that having $60 \mathrm{~min} /$ day more MVPA was associated with a 1.2 unit higher CPF score. BST was not related to CPF score independent of MVPA and ST $(\beta=0.02$, $p<0.074)$. There was an interaction effect of MVPA on the relationship between ST and CPF score (ST*MVPA $\beta=0.0004, p<0.001$ ), indicating that the relationship between ST and CPF score was moderated by MVPA. At values of $<36.30 \mathrm{~min} /$ day of MVPA, the relationship between ST and CPF score was negative (Figure 1). Between an MVPA of $\geq 36.30$ and $<107.78 \mathrm{~min} /$ day, ST was not significantly related to CPF score. At an MVPA $\geq 107.78 \mathrm{~min} /$ day, ST had a positive relationship with
CPF score. No significant interaction effect of BST on the relationship between ST and CPF score was observed $(p>0.05)$.

\section{DISCUSSION}

Our investigation suggests that higher amounts of ST were related to lower values of physical independence, regardless of time spent in MVPA and BST. However, the nature of the relationship between ST and physical independence became non-significant when older adults engaged in $\geq 36 \mathrm{~min} /$ day of MVPA. No moderation effect was observed for BST on the relationship between ST and physical independence.

Our finding that the negative relationship between ST and physical independence was independent of MVPA is similar to findings reported in the literature for other health related outcomes in older adults. ${ }^{3,4,16}$ These findings, however, do not answer the question as to whether higher amounts of MVPA can eliminate these negative relationships. Indeed, the new World Health Organization PA guidelines noted the emerging evidence on the interaction between MVPA and ST and emphasized the benefits of performing more than the recommended levels of MVPA to help reduce the detrimental effects of high amounts of sedentary behavior. ${ }^{15}$

In this investigation, we have shown that performing $36 \mathrm{~min} /$ day of MVPA can offset the negative effects of ST on an older adult's physical independence. Other studies in older adults have also come to similar conclusions regarding the moderating effect of MVPA on the relationship of ST with frailty ${ }^{10}$ and mortality. ${ }^{11,17-19}$ Mañas et al. ${ }^{10}$ found that $27 \mathrm{~min} /$ day of MVPA eliminated the negative association between ST and frailty in older adults. Although we used a similar statistical approach and our samples had similar characteristics in terms of age, sedentary time (491.4 $\mathrm{min} /$ day from our study vs. $538.7 \mathrm{~min} /$ day from Mañas et al. $^{10}$ ), and levels of MVPA (median: $9.3 \mathrm{~min} /$ day or mean: $17.1 \mathrm{~min} /$ day vs. mean: $19.7 \mathrm{~min} /$ day from Mañas et al. ${ }^{10}$ ), Mañas et al. ${ }^{10}$ found a lower cutpoint compared to ours which may be related to the different outcomes assessed. Considering the outcome of all-cause mortality, Ekelund et al. reported that $60 \mathrm{~min} /$ day of moderate $\mathrm{PA}^{17}$ or 30-40 min/day of $\mathrm{MVPA}^{11}$ were needed to attenuate the adverse association of sedentary behavior using subjective and objective PA data, respectively. Overall, based on our results and those of Mañas et al. ${ }^{10}$ and Ekelund et al., ${ }^{11,17}$ it seems that the amount of MVPA needed to offset the harmful effects of ST depends highly on the health measure examined, as well as on the assessment method for acquiring PA information. 
T A B LE 1 Descriptive characteristics of the sample of Portuguese older adults ( $\geq 65$ years old) from a national survey examining a representative sample of the Portuguese population collected between March 2017 and November 2018

$\begin{array}{lr}\text { Characteristic } & \boldsymbol{n = 8 2 1} \\ \text { Age, } \mathrm{y} & 75.5 \pm 7.4 \\ \text { Sex, } n(\%) & 254(30.9) \\ \quad \text { Male } & 567(69.1) \\ \text { Female } & \end{array}$

Education, $n(\%)^{\mathrm{a}}$

$<4$ th grade education

Up to 4th grade education

Up to 12th grade education

Higher level education (bachelors, masters, doctorate)

$63(7.7)$

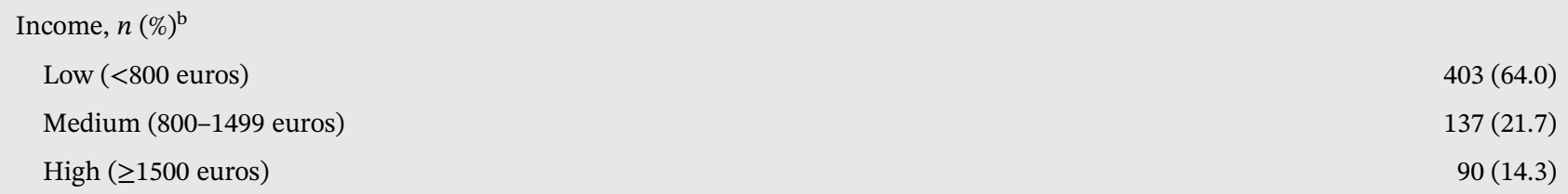

High ( $\geq 1500$ euros)

Marital status, $n(\%)^{\mathrm{c}}$

Married

Separated/divorced

Widowed

De facto union

Single

History of chronic disease, $n(\%)^{\mathrm{d}}$

Diabetes mellitus

Cancer

Cardiac Infarction

Hypertension

BMI

$\mathrm{CPF}$ score, integer value ranging from 3 to 24

Sedentary time, min/day

$491.7 \pm 103.2$

Breaks of sedentary time, number/day

$78.4 \pm 16.1$

Light-intensity physical activity, min/day

$274.2 \pm 105.0$

Moderate physical activity, min/day

$9.3(22)^{\mathrm{e}}$

Vigorous physical activity, min/day

$0(0)^{\mathrm{e}}$

Moderate-to-vigorous physical activity, min/day

Total accelerometry wear time, min/day

$771.1 \pm 77.9$

Note: total accelerometry wear time refers to the total amount of valid time the participant wore the accelerometer per valid day, with periods of at least 60 consecutive minutes of 0 counts being considered as non-wear time and a valid day consisting of daily wear time $>600$ min. Data from a national survey examining a representative sample of the Portuguese population collected between March 2017 and November 2018.

Abbreviation: $\mathrm{CPF}$, composite physical fitness score.

${ }^{\mathrm{a}} n=792$.

${ }^{\mathrm{b}} n=630$

${ }^{c} n=738$.

${ }^{\mathrm{d}} n=705$.

${ }^{\mathrm{e}}$ Median (IQR).

Interestingly, we observed that beyond $~ 108$ min/day of MVPA, ST was positively related with an older adult's ability to be physically independent. This finding is potentially related with certain benefits specific domains 
T A B LE 2 Results of multiple linear regression showing the joint associations of sedentary time (min/day), breaks in sedentary time (number/day), and moderate-to-vigorous physical activity (min/day) with CPF score adjusted for age, sex, BMI, and total accelerometer valid wear time

\begin{tabular}{lccc} 
CPF score & $\boldsymbol{\beta}$ (SE) & 95\% CI & $\boldsymbol{p}$ value \\
\hline Sedentary time & $-0.012(0.002)$ & $-0.016,-0.009$ & $<0.001$ \\
Moderate-to-vigorous physical activity & $0.021(0.008)$ & $0.005,0.037$ & $<0.008$ \\
\hline Breaks in sedentary time & $0.024(0.013)$ & $-0.002,0.049$ & 0.074 \\
\hline
\end{tabular}

Note: $\beta=$ unstandardized beta adjusted for age, sex $(0=$ female, $1=$ male), BMI, and total accelerometer wear time ( $\min /$ day). Abbreviation: $\mathrm{CPF}$, composite physical function score.

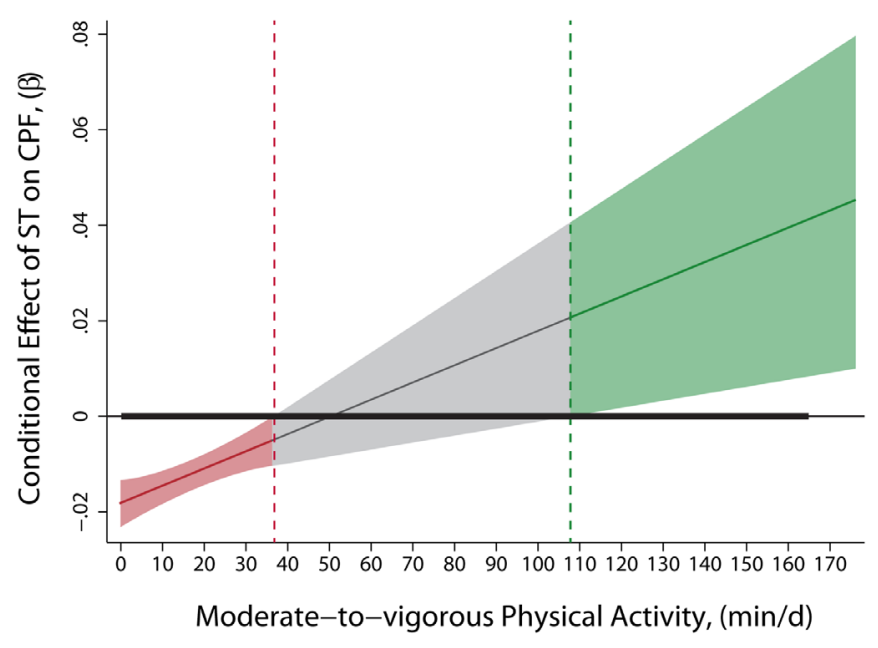

F I G U RE 1 Conditional effect of ST on CPF as a function of MVPA with regions of significance determined using the JohnsonNeyman technique. The dashed red vertical line (MVPA $=36.30 \mathrm{~min} /$ day) represents the point where the relationship between CPF and ST transitions from being significant and inversely related to being non-significant. The green dashed vertical line represents the point where the relationship between CPF and ST transitions from being non-significant to significantly positive (MVPA $=107.78 \mathrm{~min} /$ day). The gray area in between the red and green dashed lines represents the range for MVPA where ST is not significantly related to CPF (MVPA $\geq 36.30$ day and $<107.78 \mathrm{~min} /$ day). The thick horizontal black line at $y=0$ indicates the range of observed values within the sample. CPF, composite physical function score; ST, sedentary time; MVPA, moderate-to-vigorous physical activity

of sedentary behavior may provide in terms of rest and recovery. In fact, a prospective cohort study observed that women who met the PA guidelines $(\geq 450$ MET $\times$ min/ week) and reported sitting the most ( $\geq 11 \mathrm{~h} /$ day) actually had a reduced mortality risk compared to the other women who met the guidelines and had less sitting time. ${ }^{18}$ However, it is important to note that only a small portion of our sample had MVPA levels $>108 \mathrm{~min} /$ day. More studies including a greater number of individuals falling within the upper end of the MVPA spectrum are needed to verify if ST can be beneficial for older adults who perform high levels of activity throughout the day.

Although BST has been found to be positively related with aspects of an older adult's functional capacity independent of MVPA, ${ }^{5-8}$ we did not observe a similar relationship between BST and an older adult's physical independence. We also observed no moderation effect of BST on the ST and physical independence relationship. Thus, although promoting increases in BST may be beneficial for certain functional abilities of older adults, ${ }^{5-8}$ results from our investigation indicate that this action does not prove to be strong enough to fully eliminate the negative effects of ST on an older adult's physical independence. Nevertheless, for the many older adults who struggle to reach the $36 \mathrm{~min} /$ day cutpoint for MVPA, and as suggested by the new PA guidelines, ${ }^{15}$ a more feasible approach to maximize the healthy aging process may be to limit the amount of time in sedentary pursuits and incorporate any incremental increase in MVPA, as these actions may still prove to be beneficial and more likely attainable for the majority of the older adult population.

A major limitation of this study is the cross-sectional nature of the analysis, which does not allow for the establishment of causality, and, therefore, we cannot exclude the existence of reverse causality in the relationships. Further studies using longitudinal approaches are warranted. Although we had a large sample of Portuguese older adults from different regions of Portugal, the majority of our sample was Caucasian (99.2\%), thus limiting the generalizability of our results to other ethnicities. Despite limitations, our use of objectively measured PA and ST from accelerometers improves upon the self-reported measurements used in previous studies. It is important to note, however, that PA outcomes can be highly influenced by how the raw accelerometer data is processed and the intensity threshold for PA intensity classification used. ${ }^{20}$ For instance, lowering the moderate PA intensity threshold to one that has been suggested to be more acceptable for older adults (i.e., 1013 counts $/ \mathrm{min}$ ), ${ }^{21}$ greatly increases the MVPA requirement (i.e., $77 \mathrm{~min} /$ day) needed to overcome the negative effects of ST on CPF. Given that for 
older adults there is no clear guideline discriminating the best PA thresholds to use, we chose the same one used by other studies ${ }^{10,11}$ assessing the moderating effects of MVPA on the relationship between ST and health related outcomes in older adults to facilitate result comparisons. It is important for researchers to take the thresholds chosen into consideration when interpreting and comparing study results.

In conclusion, MVPA moderated the relationship between ST and an older adult's physical independence, such that performing $>36 \mathrm{~min} /$ day of MVPA eliminated the negative association of ST. Moreover, performing $>108 \mathrm{~min} /$ day of MVPA promoted a positive association between ST and physical independence, indicating that for highly active older adults, ST may be beneficial for one's ability to maintain physical independence. For older adults who struggle to perform sufficient amounts of any type of PA, decreasing ST and increasing MVPA by any incremental amount should not be overlooked as a viable strategy to improve healthy aging.

\section{ACKNOWLEDGMENTS}

\section{FINANCIAL DISCLOSURE}

This investigation was funded by the Portuguese Foundation for Science and Technology (UIDB/00447/2020). IRC is supported by the Portuguese Foundation for Science and Technology (IRC: SFRH/BD/149394/2019).

\section{CONFLICT OF INTEREST}

The authors have no conflicts.

\section{AUTHOR CONTRIBUTIONS}

João P. Magalhães, Megan Hetherington-Rauth, Asier Mañas, Ignacio Ara, and Luís B. Sardinha contributed to the conception and design of the study. João P. Magalhães, Pedro B. Júdice, Gil B. Rosa, Inês R. Correia were responsible for data collection and acquisition. Megan Hetherington-Rauth was responsible for data analysis and interpretation. Megan Hetherington-Rauth and João P. Magalhães drafted the manuscript. Luís B. Sardinha, Pedro B. Júdice, Ignacio Ara, Asier Mañas, and Inês R. Correia contributed to reviewing and editing the manuscript. All authors approved the final version of the manuscript and take responsibility for the integrity of the data and the accuracy of the data analysis.

\section{SPONSOR'S ROLE}

None.

\section{ORCID}

Asier Mañas (1) https://orcid.org/0000-0002-1683-1365 Luís B. Sardinha (D) https://orcid.org/0000-0002-6230-6027

\section{REFERENCES}

1. Moreno-Agostino D, Daskalopoulou C, Wu YT, et al. The impact of physical activity on healthy ageing trajectories: evidence from eight cohort studies. Int J Behav Nutr Phys Act. 2020;17:92.

2. Taylor D. Physical activity is medicine for older adults. Postgrad Med J. 2014;90:26-32.

3. Copeland JL, Ashe MC, Biddle SJ, et al. Sedentary time in older adults: a critical review of measurement, associations with health, and interventions. Br J Sports Med. 2017;51:1539.

4. Mañas A, Del Pozo-Cruz B, García-García FJ, GuadalupeGrau A, Ara I. Role of objectively measured sedentary behaviour in physical performance, frailty and mortality among older adults: a short systematic review. Eur J Sport Sci. 2017;17: 940-953.

5. Chen T, Narazaki K, Haeuchi Y, Chen S, Honda T, Kumagai S. Associations of sedentary time and breaks in sedentary time with disability in instrumental activities of daily living in community-dwelling older adults. J Phys Act Health. 2016;13: 303-309.

6. Sardinha LB, Santos DA, Silva AM, Baptista F, Owen N. Breaking-up sedentary time is associated with physical function in older adults. J Gerontol A Biol Sci Med Sci. 2015;70: 119-124.

7. Sardinha LB, Ekelund U, dos Santos L, Cyrino ES, Silva AM, Santos DA. Breaking-up sedentary time is associated with impairment in activities of daily living. Exp Gerontol. 2015;72: $57-62$

8. Davis MG, Fox KR, Stathi A, Trayers T, Thompson JL, Cooper AR. Objectively measured sedentary time and its association with physical function in older adults. $J$ Aging Phys Act. 2014;22:474-481.

9. Mañas A, Del Pozo-Cruz B, Rodríguez-Gómez I, et al. Breaking sedentary time predicts future frailty in inactive older adults: a cross-lagged panel model. J Gerontol A Biol Sci Med Sci. 2020;glaa159. https://doi.org/10.1093/gerona/glaa159.

10. Mañas A, Pozo-Cruz BD, Rodríguez-Gómez I, et al. Can physical activity offset the detrimental consequences of sedentary time on frailty? A moderation analysis in 749 older adults measured with accelerometers. J Am Med Dir Assoc. 2019;20: 634-638.e631.

11. Ekelund U, Tarp J, Fagerland MW, et al. Joint associations of accelero-meter measured physical activity and sedentary time with all-cause mortality: a harmonised meta-analysis in more than 44000 middle-aged and older individuals. $\mathrm{Br} J$ Sports Med. 2020;54:1499-1506.

12. Lohman TG, Roche AF, Martorell R. Anthropometric Standardization Reference Manual. Champaign, IL: Human Kinetics Books; 1988.

13. Rikli RE, Jones CJ. The reliability and validity of a 6-minute walk test as a measure of physical endurance in older adults. J Aging Phys Act. 1998;6:363.

14. Troiano RP, Berrigan D, Dodd KW, Masse LC, Tilert T, McDowell M. Physical activity in the United States measured by accelerometer. Med Sci Sports Exerc. 2008;40:181-188.

15. Bull FC, Al-Ansari SS, Biddle S, et al. World Health Organization 2020 guidelines on physical activity and sedentary behaviour. Br J Sports Med. 2020;54:1451-1462.

16. Chau JY, Grunseit AC, Chey T, et al. Daily sitting time and allcause mortality: a meta-analysis. PLoS One. 2013;8:e80000. 
17. Ekelund U, Steene-Johannessen J, Brown WJ, et al. Does physical activity attenuate, or even eliminate, the detrimental association of sitting time with mortality? A harmonised metaanalysis of data from more than 1 million men and women. Lancet. 2016;388:1302-1310.

18. Pavey TG, Peeters GG, Brown WJ. Sitting-time and 9-year allcause mortality in older women. Br J Sports Med. 2015;49:95-99.

19. Ensrud KE, Blackwell TL, Cauley JA, et al. Objective measures of activity level and mortality in older men. J Am Geriatr Soc. 2014;62:2079-2087.

20. Migueles JH, Cadenas-Sanchez C, Ekelund U, et al. Accelerometer data collection and processing criteria to assess physical activity and other outcomes: a systematic review and practical considerations. Sports Med. 2017;47:1821-1845.
21. Barnett A, van den Hoek D, Barnett D, Cerin E. Measuring moderate-intensity walking in older adults using the ActiGraph accelerometer. BMC Geriatr. 2016;16:211.

How to cite this article: Hetherington-Rauth $M$, Magalhães JP, Júdice PB, et al. Physical activity moderates the effect of sedentary time on an older adult's physical independence. J Am Geriatr Soc. 2021;69:1964-1970. https://doi.org/10.1111/jgs. 17141 